\title{
Variaciones Morfométricas de la Próstata de Chinchilla laniger (MOLINA, 1982) y de la Concentración de Testosterona Plasmática Durante un Ciclo Reproductivo Anual
}

\author{
Morphometric Variations of Chinchilla laniger Prostate and Plasmatic \\ Testosterone Concentration During its Annual Reproductive Cycle \\ Raquel Cepeda C.; Luis Adaro A. \& Paola Peñailillo G.
}

CEPEDA, C. R.; ADARO, A. L. \& PEÑAILILLO, G. P. Variaciones morfométricas de la próstata de Chinchilla laniger (MOLINA, 1982) y de la concentración de testosterona plasmática durante un ciclo reproductivo anual. Int. J. Morphol., 24(1):89-97, 2006.

RESUMEN: Durante un año, se estudió la próstata de individuos adultos de la especie Chinchilla laniger (MOLINA, 1982), mantenidos en cautiverio, para detectar posibles variaciones estacionales en el peso glandular y corporal, índice órgano-somático (IOS), grosor de la pared y altura del epitelio glandular y concentración de testosterona plasmática. Además, se realizó un análisis histoquímico con técnicas de PAS, diastasa-PAS y Azul de Alcian $\mathrm{pH} 1$ y $\mathrm{pH}$ 2,5.

Se describió una Zona A, periférica y una Zona B, central. En ambas, las células epiteliales siempre fueron negativas al Azul de Alcian. En la Zona A, se detectó la presencia de glicoproteínas neutras con intensa reacción PAS (+++) entre octubre y abril y leve a moderada $(+/++)$ entre mayo y septiembre, y ausencia de glicógeno. La Zona B siempre fue PAS negativa. Los valores morfométricos máximos se registraron entre mayo y julio y los mínimos entre noviembre y febrero, presentando diferencias estacionales significativas ( $\leq \leq 0,05)$, con excepción del peso corporal, que no mostró variaciones durante el año. La concentración de testosterona plasmática presentó los valores mínimos en enero, febrero y junio y los máximos en abril, mayo, julio y agosto.

Se concluye, que la próstata de la Chinchilla laniger en cautiverio, presenta la mayor actividad secretora durante el período otoño-invierno, respondiendo positivamente a la concentración de testosterona y coincidiendo con la máxima actividad reproductiva descrita para la especie en el mismo periodo.

PALABRAS CLAVE: Próstata; Chinchilla laniger; Estacionalidad reproductiva; Roedor.

\section{INTRODUCCIÓN}

La Chinchilla laniger (MOLINA, 1982) es un roedor autóctono de la Cordillera de Los Andes del Perú, Chile, Bolivia y Argentina. Es una especie endémica de Chile (Spotorno \& Walker, 2000; Spotorno et al., 2004), clasificada como en peligro de extinción (Thornback \& Jenkis, 1982; Glade, 1988) y con una alta prioridad de conservación dentro de los mamíferos chilenos (Cofré \& Marquet, 1999). En Chile, sólo existen poblaciones silvestres en la zona de Aucó, al norte de Illapel y en Quebrada Honda (La Higuera), al norte de la Serena (Campos, 1996), que fluctúan alrededor de los 11.000 individuos (Jiménez, 1995) y con una clara tendencia a disminuir. Por otra parte, se ha informado la existencia de alrededor de 8600 ejemplares en confinamiento en 56 criaderos (SAG, 1996), animales que han permitido la realización de diversas investigaciones y que, además, podrían constituir una importante reserva para el repoblamiento, en caso de disminuir a niveles críticos las poblaciones silvestres.

Se ha descrito que los animales mantenidos en confinamiento presentan un peso corporal que fluctúa entre 500 y 600 gramos, tienen una gestación de 111 días y viven alrededor de 10 a 12 años (Parodi, 1990).

Al estado silvestre, esta especie se reproduce en los meses de invierno (Weir, 1972; Neira, 1987; Rodríguez, 1988; García et al., 1989; Neira et al., 1989; Marinovic, 1990), estacionalidad reproductiva que está asociada con 
cambios endocrinos, estructurales y funcionales de los órganos reproductivos, entre ellos las glándulas sexuales anexas, las que se ha demostrado son andrógeno-dependientes (Carballada et al., 1995).

La próstata es una glándula impar, que se encuentra en todos los mamíferos domésticos (Dellmann, 1993), presentando variaciones morfológicas en las distintas especies (Miller et al., 1965; Reighardt \& Jennings, 1966; Holtz \& Foote, 1978; Hayashi et al., 1991; Kinbara \& Cunha, 1996 y Vásquez \& del Sol, 2002). La secreción prostática contribuye al volumen total del eyaculado y contiene iones citrato, calcio y fósforo, enzimas hidrolíticas, profibrinolisina, glucógeno, mucosustancias neutras, sialomucinas y algunas proteínas no catalíticas (Moussa et al., 1983; Vanha-Pertula et al., 1990; Martinelli et al., 1991). Una de sus funciones es neutralizar el plasma seminal, que se acidifica por la acumulación de dióxido de carbono metabólico y lactato, permitiendo neutralizar la acidez de la secreción vaginal, lo que facilita la motilidad y viabilidad de los espermatozoides en el tracto femenino (Dellmann).

La información sobre la próstata de este roedor y su patrón de comportamiento en relación con la actividad reproductiva de la especie es escasa, por lo que el propósito de este trabajo fue describir, a través de un año, algunos aspectos anatómicos e histológicos de la glándula, asociados a variaciones en la concentración de testosterona plasmática.

\section{MATERIAL Y MÉTODO}

Se utilizaron 50 machos, en edad reproductiva (2 a 3 años de edad), clínicamente sanos y mantenidos en cautiverio bajo similares condiciones de manejo: temperatura ambiente, luz natural, alimentación sobre la base de pellets y heno de alfalfa ad libitum. Durante un año, se sacrificó un promedio de 4 animales al mes, con sobredosis de tiopental sódico al 2,5\%, administrado por vía intraperitoneal.

Registros morfométricos macroscópicos: se registró el peso corporal y glandular (g) de cada animal y se determinó el índice órgano-somático (IOS), de acuerdo a la siguiente fórmula:

$$
\text { IOS }(\%)=\text { Peso órgano }(\mathrm{g}) / \text { Peso corporal }(\mathrm{g}) \times 100
$$

Histología e histoquímica: Las próstatas de todos los animales fueron fijadas en Bouin-Holland y procesadas para inclusión en paraplast. Se hicieron cortes transversales de $5 \mu \mathrm{m}$ de grosor que se tiñeron con Hematoxilina y Eosina (HE), Tricrómico de van Gieson (vG), Ácido Peryódico Schiff (PAS) y Azul de Alcian a pH 1 y pH 2,5 (López et al., 1982). Como control para glicógeno, se hizo digestión con diastasa, para lo cual los cortes se incubaron por 60 minutos, a $37^{\circ} \mathrm{C}$, en cámara húmeda, previo a la tinción con PAS. Las reacciones con PAS y Azul de Alcian se calificaron como: negativas (-), levemente teñidas (+), moderadamente teñidas $(++)$ e intensamente teñidas $(+++)$. Los cortes histológicos fueron observados y fotografiados en un microscopio Nikon, modelo Eclipse 600, con videocámara incluida.

\section{Registros morfométricos microscópicos:}

Grosor de la pared glandular: se midieron 10 cortes por glándula, con aumento de $10 \mathrm{X}$, en cuatro puntos correspondientes a las alturas máximas de la pared, sin incluir la mucosa. Dada la morfología de la próstata, esta medición se realizó en la zona periférica, que se denominó zona A (ZA) y en la zona central o zona B (ZB). Se obtuvo el promedio mensual de los animales analizados.

Altura del epitelio secretor: con un aumento de 100X, se midió la altura del epitelio en las mismas zonas descritas en el punto anterior. Luego se obtuvo el promedio mensual.

Determinación de testosterona plasmática: Previo al sacrificio de los animales, se obtuvo sangre periférica del pabellón auricular con capilares de microhematocrito heparinizado. La sangre se centrifugó a 1200 g, durante 10 minutos en microcentrífuga refrigerada, y el plasma se conservó a $-24^{\circ} \mathrm{C}$ hasta su procesamiento mediante radioinmunoensayo (RIA) de fase sólida DPC (Diagnostic Product Corporation, Los Angeles CA, USA). Las determinaciones se hicieron por triplicado, previa validación del método para chinchilla.

Análisis estadístico: Los valores obtenidos en los registros morfométricos macroscópicos y microscópicos se describieron a través de la media aritmética y la desviación típica. Además, se determinó la significancia estadística de los resultados mediante un análisis de varianza anidado, empleando el paquete estadístico SAS. Se calcularon coeficientes de correlación de Pearson (r) entre las variables.

Los valores de las concentraciones de testosterona plasmática se expresaron a través de la media aritmética y la desviación típica y se usó análisis de varianza y la prueba de Tukey para comparar los valores entre los meses estudiados. Los resultados fueron tabulados y graficados. 


\section{RESULTADOS}

La observación macroscópica muestra, que la próstata es un órgano impar que se sitúa caudal a las vesículas seminales y conductos deferentes, los que llegan en forma independiente a la uretra. Hacia craneal se relaciona con la vejiga urinaria y hacia caudal con la uretra pelviana. Presenta dos lóbulos laterales de aspecto difuso y un cuerpo central que rodea a la uretra intrapélvica, lo que le confiere forma de mariposa, y se encuentra cubierta por una fina envoltura de tejido conectivo laxo que no constituye una cápsula definida.

\section{Registros morfométricos macroscópicos.}

Peso Corporal: En la Tabla I se puede ver, que el promedio mensual más alto se obtuvo en septiembre $(570,6 \pm 23,28 \mathrm{~g})$ y el más bajo en junio $(456,1 \pm 47,79 \mathrm{~g})$. No se encontraron diferencias significativas entre los meses estudiados $(\mathrm{p} \geq 0,05)$.

Peso de la próstata: Como se muestra en la Tabla I, el promedio mensual más alto se registró en julio $(1,52 \pm 0,279$ $\mathrm{g})$, mientras que el más bajo se obtuvo en enero $(0,553 \pm$ $0,149 \mathrm{~g}$ ), siendo significativamente menor que los valores obtenidos en mayo, julio y octubre $(\mathrm{p} \leq 0,05)$. El análisis de correlación entre peso prostático y peso corporal mostró un $r=0,1281$, no significativo.

Tabla I. Peso corporal (PC; g), Peso próstata (PP, g), IOS (\%) en chinchillas (Chinchilla laniger). Promedios \pm desviaciones típicas mensuales.

\begin{tabular}{|c|c|c|c|c|c|c|c|}
\hline MES & PC & PP & IOS & MeS & PC & PP & IOS \\
\hline ENE & $520,4 \pm$ & $0,553 . \pm 0,149_{\mathrm{C}}$ & $0,105 \pm 0,022_{\mathrm{c}}$ & $J U L$ & $524,6 \pm$ & $1,520 \pm 0,279_{\mathrm{A}}$ & $0,291 \pm 0,053_{\mathrm{A}}$ \\
\hline$F E B$ & $497,4 \pm$ & $0,685 \pm 0,406_{\mathrm{B}}$ & $0,146 \pm 0,102_{\mathrm{A}}$ & $A G O$ & $489,5 \pm$ & $1,093 \pm 0,203_{\mathrm{A}}$ & $0,226 \pm 0,055_{\mathrm{A}}$ \\
\hline$M A R$ & $561,0 \pm$ & $0,797 \pm 0,493_{\text {в }}$ & $0,151 \pm 0,103_{\mathrm{A}}$ & SEP & $570,6 \pm$ & $0,895 \pm 0,312_{\text {в, }}$ & $0,155 \pm 0,048_{\mathrm{A},}$ \\
\hline ABRIL & $550,3 \pm$ & $0,662 \pm 0,375_{\mathrm{B}}$ & $0,123 \pm 0,078_{\mathrm{C}}$ & $O C T$ & $503,8 \pm$ & $1,316 \pm 0,055_{\mathrm{A}, \mathrm{B}}$ & $0,264 \pm 0,035_{\mathrm{A}}$ \\
\hline MAY & $556,2 \pm$ & $1,271 \pm 0,303_{\mathrm{A}}$ & $0,230 \pm 0,061_{\mathrm{A}}$ & $\mathrm{NoV}$ & $470,3 \pm$ & $0,799 \pm 0,352_{\text {в, }}$ & $0,169 \pm 0,072_{\mathrm{A},}$ \\
\hline$J U N$ & $456,1 \pm$ & $0,785 \pm 0,167_{\mathrm{B}}$ & $0,180 \pm 0,067_{\mathrm{A}}$ & DIC & $477,2 \pm$ & $0,772 \pm 0,134_{\mathrm{B}, \mathrm{C}}$ & $0,163 \pm 0,034_{\mathrm{A},}$ \\
\hline
\end{tabular}

Letras en subíndice distintas indican diferencias significativas $(\mathrm{p} \leq 0,05)$

IOS: El valor más alto fue en julio $(0,291 \pm 0,053 \%)$ y los mínimos en enero y abril $(0,105 \pm 0,022 \%$ y $0,123 \pm 0,078 \%$, respectivamente), siendo estas diferencias estadísticamente significativas $(\mathrm{p} \leq 0,05)$ (Tabla I). El coeficiente de correlación calculado entre el IOS y el peso de la glándula, dio un valor para $\mathrm{r}=0,9714$, que resultó muy significativo ( $\mathrm{p} \leq$ 0,0001) (Tabla II).
Tabla II. Coeficientes de correlación de Pearson (r) y niveles de significancia (p)

\begin{tabular}{llcc}
\hline \multicolumn{2}{c}{ VARIABLES } & $\mathrm{R}$ & $\mathrm{P}$ \\
\hline IOS PRÓSTATA & - PESO PRÓSTATA & 0,9714 & $<0,0000$ \\
GROSOR PAREDZONA A & - GROSOR PAREDZONA B & 0,9243 & $<0,0000$ \\
AL TURA BPITELIO ZONA A & - GROSOR PAREDZONA A & 0,9498 & $<0,0000$ \\
AL TURA EPITELIO ZONAB & - GROSOR PAREDZONA B & 0,9564 & $<0,0000$ \\
AL TURA EPITELIO ZONA A & - ALTURA EPITELIOZONA B & 0,9602 & $<0,0000$ \\
\hline
\end{tabular}




\section{Características histológicas e histoquímicas.}

Zona A (ZA): se caracterizó por la presencia de adenómeros alveolares de lumen amplio y regular, formados por epitelio simple y una envoltura de tejido conectivo laxo y músculo liso (Fig. 1). Entre los meses de septiembre y abril, las células epiteliales fueron cúbicas, con núcleos centrales que mostraban numerosos gránulos de cromatina distribuídos uniformemente y 1 o 2 nucléolos. En cambio, entre mayo y agosto el epitelio aumentó de altura y pasó a ser prismático, con núcleos en el tercio basal de las células y con gránulos de cromatina que tendieron a concentrarse alrededor de la membrana nuclear. Sólo esta zona fue PAS positiva, cuya intensidad mostró variaciones en los meses analizados. Así, entre septiembre y marzo, se observaron algunos adenómeros con células epiteliales PAS (++) en la zona apical, alternadas con células (-) y con secreción en el lumen PAS (++/+++). Otros adenómeros fueron PAS (-) tanto para la secreción, como para las células epiteliales. Entre abril y agosto, la intensidad de la reacción fue menor en los adenómeros PAS positivos, persistiendo células PAS (-) y PAS (+/++) en su zona apical, con secreción PAS (++) en el lumen. Los controles diastasa-PAS fueron siempre positivos.

Zona B (ZB): a diferencia de la anterior, esta zona se caracterizó porque los adenómeros alveolares presentaron una envoltura de tejido conectivo laxo y músculo liso de mayor desarrollo, y porque la mucosa presentó proyecciones largas hacia el lumen, dándole un aspecto irregular y más estrecho (Fig. 1). El epitelio prismático monoestratificado de estas proyecciones, siempre fue PAS (-). Los adenómeros de las dos zonas prostáticas descritas, fueron negativos a la reacción de Azul de Alcian pH 1 y pH 2,5 en todos los meses observados.

\section{Registros morfométricos microscópicos.}

Grosor de la pared de los adenómeros de la ZA: Los promedios mensuales se incrementaron de febrero a julio, registrándose los valores máximos en junio y julio $(26,17$ $\pm 0,367 \mathrm{~mm}$ y $27,22 \pm 1,238 \mathrm{~mm}$, respectivamente) y los mínimos en enero y febrero $(7,69 \pm 0,089 \mu \mathrm{m}$ y $8,48 \pm$ $0,071 \mathrm{~mm}$, respectivamente). Las diferencias obtenidas entre todos los meses de invierno y los de verano, resultaron estadísticamente significativas $(\mathrm{p} \leq 0,05)$ (Gráfico I).

Grosor de la pared de los adenómeros de la ZB: El comportamiento de esta variable fue similar al observado en la ZA (Gráfico I). Los promedios mensuales más altos fueron en el período mayo-julio, presentando el valor máximo julio $(39,85 \pm 0,508 \mu \mathrm{m})$, y los más bajos entre diciembre y febrero, siendo el mínimo en enero $(16,1632 \pm 0,14212$ $\mu \mathrm{m})$. Las diferencias entre todos los meses fueron significativas $(p \leq 0,05)$. El coeficiente de correlación calculado entre el grosor de la pared de ambas zonas prostáticas (Tabla II) dio un valor de $r=0,9243$, muy significativo $(p \leq 0,0001)$.

Altura del epitelio secretor ZA: En el Gráfico II, se ve que los valores máximos correspondieron al período mayoagosto y los mínimos a diciembre-febrero. El promedio mensual más alto fue en julio $(18,17 \pm 0,221 \mu \mathrm{m})$ y el más bajo en enero $(4,92 \pm 0,006 \mu \mathrm{m})$. Las diferencias encontradas entre los promedios de todos los meses del período analizado resultaron significativas $(\mathrm{p} \leq 0,05)$, excepto para enero y febrero, que fueron similares.

Altura del epitelio secretor ZB: Los valores más altos se presentaron en junio y julio $(18,88 \pm 0,431 \mu \mathrm{m}$ y $18,79 \pm$ $0,123 \mu \mathrm{m}$, respectivamente) y el mínimo $(8,49 \pm 0,086 \mu \mathrm{m})$ en enero (Gráfico II). Las diferencias entre los períodos extremos fueron significativas $(\mathrm{p} \leq 0,05)$. El coeficiente de correlación, entre la altura del epitelio de ambas zonas, fue altamente significativo $(r=0,9602, p \leq 0,0001)$. Este coeficiente también fue significativo entre la altura del epitelio y el grosor de la pared respectiva (Tabla II).

Concentración de testosterona plasmática. Como se muestra en el Gráfico III, los valores hormonales mínimos se registraron durante enero, febrero y junio $(1,39 \pm 0,34$, $1,0 \pm 0,22$ y $1,17 \pm 0,51 \mathrm{nmol} / \mathrm{L}$, respectivamente) y los máximos en abril, mayo, julio y agosto. Al analizar el comportamiento anual de la hormona, se observó una curva bimodal que, en general, coincide con el IOS obtenido durante el mismo periodo (Gráfico III) y con la altura del epitelio en ambas zonas (Gráfico II).

\section{DISCUSIÓN}

La morfología macroscópica de la próstata de chinchilla, es semejante a la descrita en el gato y perro (Miller et al.; Reighardt \& Jennings), con dos lóbulos unidos por un istmo, pero difiere de lo informado para otros roedores como la rata, que presenta 4 lóbulos (Hayashi et al.; Kinbara \& Cunha) y el conejo, en el que se describe un complejo prostático constituido por tres porciones: propróstata, próstata y parapróstata, que presentan claras diferencias anatómicas, histológicas e histoquímicas, pues cumplirían roles distintos en el proceso reproductivo (Holtz \& Foote; Vásquez \& del Sol). Así también, el tejido conectivo laxo que rodea a la próstata no constituye una cápsula definida, otorgándole a la glándula un aspecto difuso, diferente a la cápsula fibromuscular que circunscribe a la próstata del conejo (Vásquez \& del Sol) y del hombre (Ayala et al., 1989; Sattar et al., 1995). 
Gráfico I. Grosor de pared: Zonas A y B / IOS

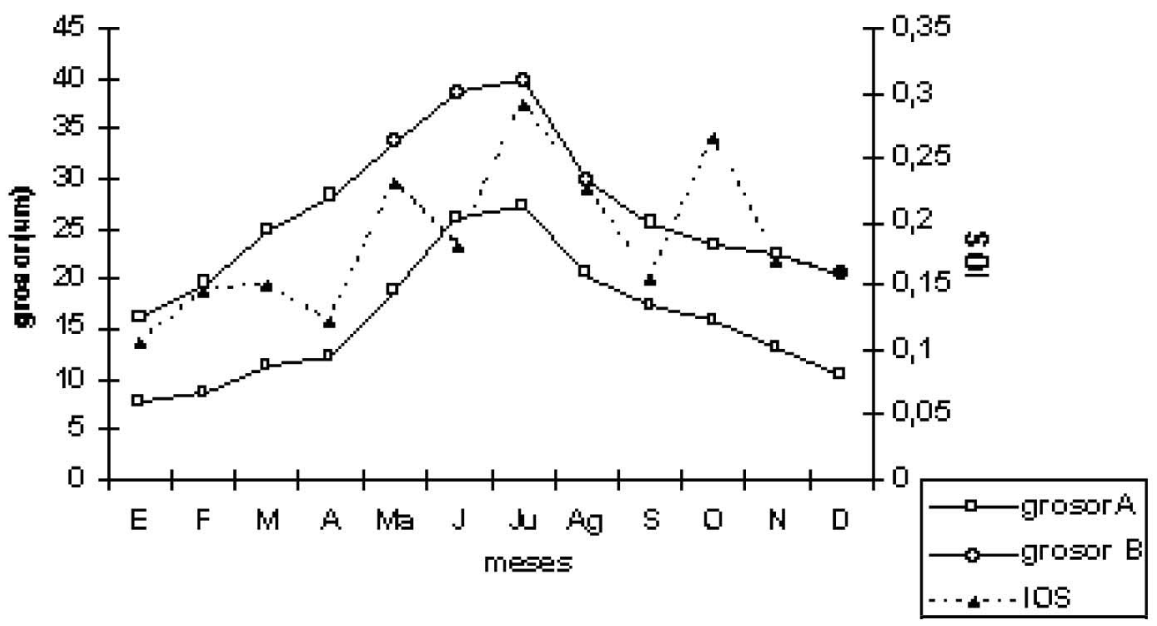

Gráfico II. Concentración de testosterona plasmática $(\mathrm{nm} / \mathrm{L})$ y altura de epitelio de zonas A y B

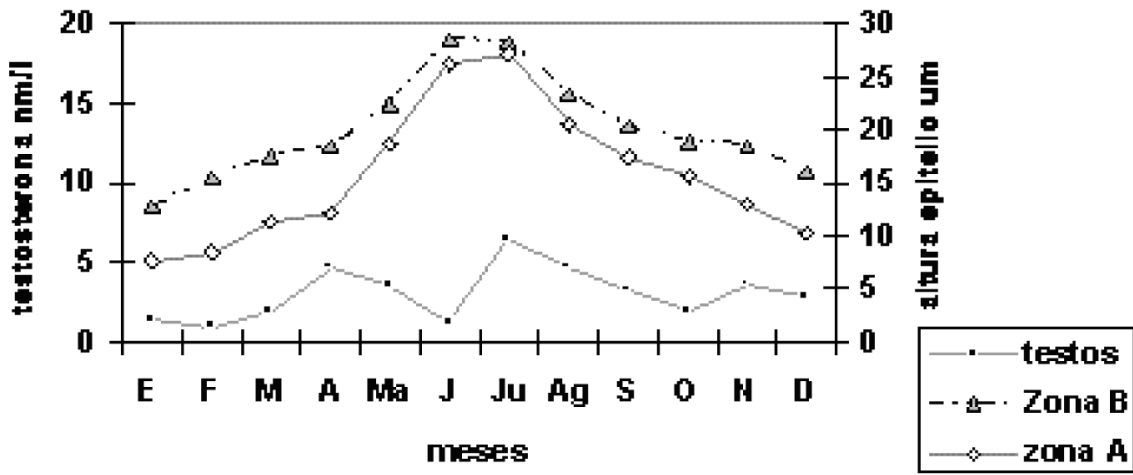

Gráfico III. Ïndice organosomático (IOS) y concentración de testosterona plasmática (nml/L)

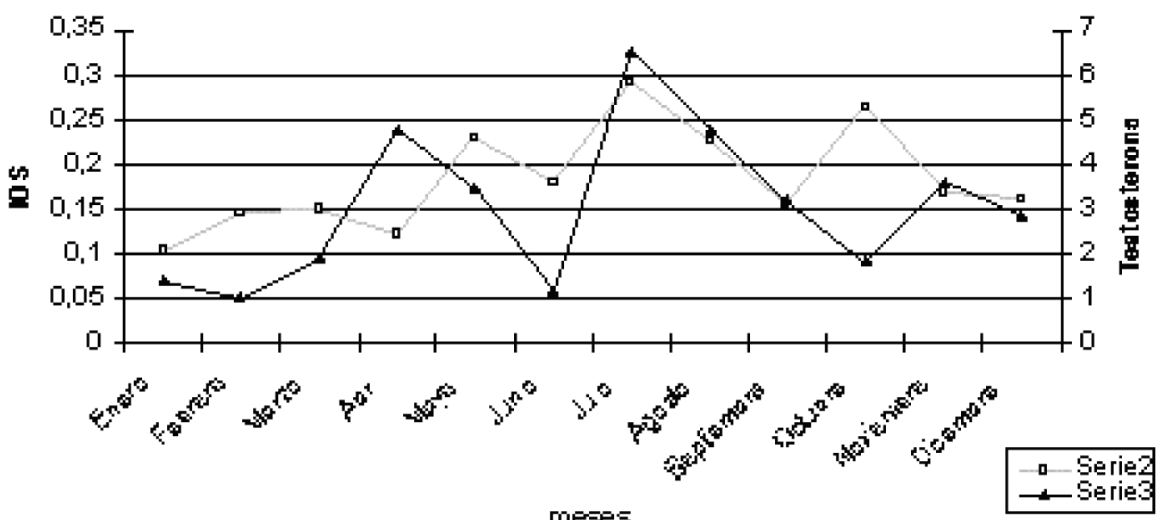

meres
Las variaciones del peso prostático a lo largo del año, no fueron significativas, sin embargo, se observó una tendencia a obtener valores más bajos entre los meses de noviembre y abril, similar a lo observado en el peso de las glándulas bulbouretrales de estos animales para los mismos meses (Cepeda et al., 1999). No obstante, hay que consignar que hubo dificultades en la disección de la glándula, debido a la ausencia de una cápsula fibrosa bien definida, pero que está en estrecha relación con los tejidos contiguos con los que, además, presenta un color similar, situación que no se observa en las glándulas bulbouretrales y que podría explicar la variabilidad observada, por pérdida de tejido prostático durante la disección. Una complicación similar fue descrita por Contreras \& BustosObregón (1977), quienes tuvieron dificultades para disecar el epidídimo de Octodon degus, libre de grasa y así determinar el peso exacto del conducto.

Los resultados obtenidos en este estudio, no arrojaron una correlación significativa entre peso corporal e IOS, sugiriendo que, por sí solos, no son buenos indicadores de variaciones estacionales en los órganos reproductivos, situación similar a lo informado por Contreras \& Bustos-Obregón en Octodon degus. Sin embargo, la alta y significativa correlación entre el IOS y el peso glandular, indicaría que este índice reflejaría mejor la actividad reproductiva que el peso corporal, que no mostró variaciones significativas durante el periodo en estudio debido, probablemente, a que los animales dispusieron de alimentación ad libitum, a diferencia de lo que sucede en condiciones naturales, donde el consumo de alimento varía según la época del año (Branch, 1993). Los valores extremos obtenidos en el IOS, indicarían que la máxima actividad 


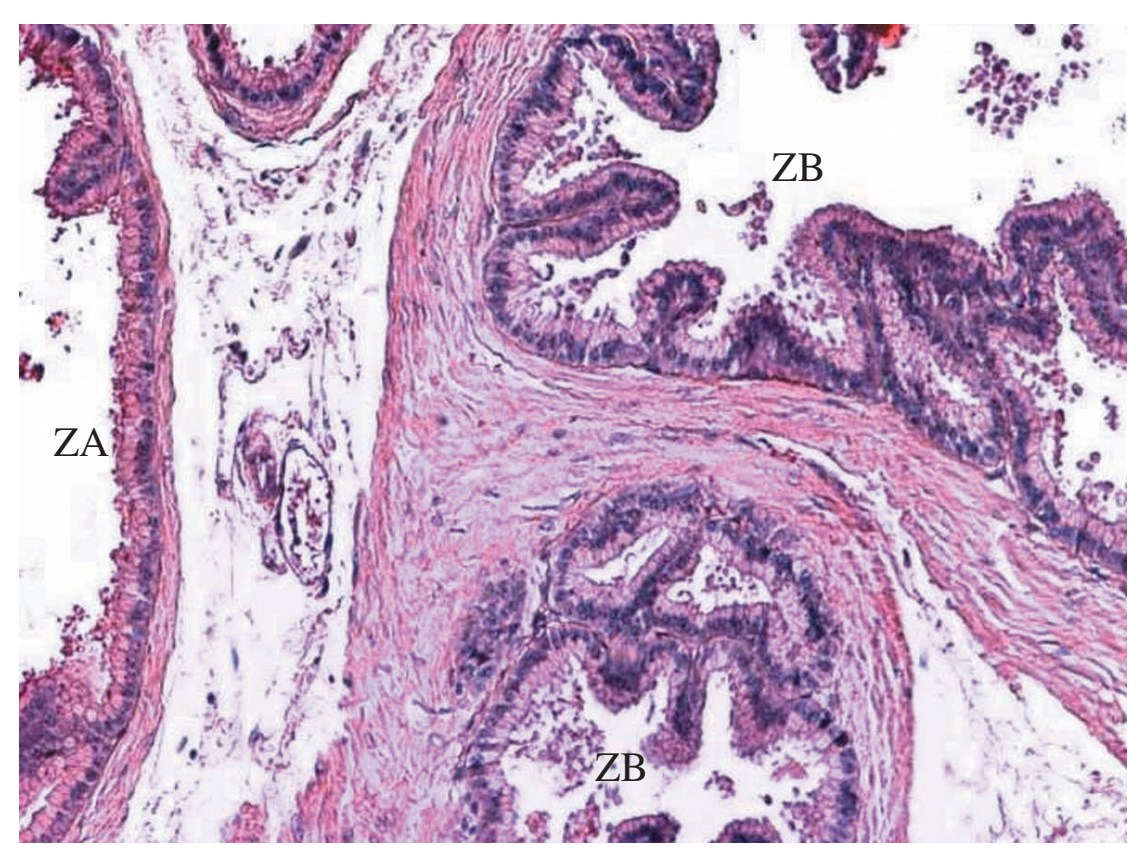

Fig. 1. Corte histológico de próstata de Chinchilla laniger, (MOLINA, 1982), correspondiente al mes de junio, donde se muestran las Zonas: A (ZA) y B(ZB). Tinción H-E. 100x.

secretora se produce en invierno y la mínima en verano, situación que es coincidente con la actividad reproductiva invernal y el reposo gonadal durante el verano, descrito para estos roedores (Adaro et al., 1999).

El mismo patrón estacional se observó en la concentración de testosterona plasmática, con valores hormonales similares a los informados por Adaro et al., (2002), quienes encontraron la mayor concentración de testosterona sérica en julio y la menor en enero, en un grupo de chinchillas en confinamiento estudiadas durante un año.

Asimismo, la tendencia estacional de la altura del epitelio a concentrar los valores máximos durante los meses invernales, se debería a una mayor actividad secretora de las células epiteliales, la que a su vez estaría en directa relación con la concentración de testosterona plasmática, coincidiendo con lo observado por Murakoshi et al. (1993) en próstata de ratas castradas y por Orgad et al., (1984) en perros castrados, quienes demostraron que el epitelio prostático aumenta de altura en respuesta a la administración de testosterona exógena (Murakoshi et al., 1992). Es así, como en la próstata canina, a partir de los 3 días post castración, es posible observar cambios morfológicos graduales, tanto en el estroma como en el epitelio. Mientras las células epiteliales cambian de cilíndricas altas a cilíndricas bajas o cuboidales, el lumen glandular y el volumen de células estromales se reducen luego de la castración (Niu et al., 2003).

Las diferencias significativas $(\mathrm{p}<0,05)$ entre los $\mathrm{pe}$ riodos extremos para la altura del epitelio, que reflejarían cambios en la actividad secretora glandular, se deberían a variaciones en la concentración de receptores de andrógenos (RA) en las células epiteliales, a los cuales debe unirse la testosterona para poder ejercer sus efectos biológicos. En la próstata de ratas adultas, las células epiteliales secretoras muestran una fuerte inmunorreactividad para RA, y la reducción de los niveles séricos y prostáticos de 5aDihidrotestosterona por castración, resultan en la pérdida de un $70 \%$ de estas células por apoptosis (Heinlein \& Chang, 2004). Así también, Prins \& Birch (1993) observaron que en la próstata ventral y dorsal de la rata, el total de RA citosólicos y nucleares alcanza muy bajos niveles dentro de los 7 días posteriores a la castración, pero retorna a niveles normales dentro de los 3 días siguientes al reemplazo de testosterona, indicando que los andrógenos, a su vez, regulan a sus receptores en estas regiones prostáticas.

Esto es consistente con lo informado en otros estudios, en los que mediante técnicas de inmunomarcación, observaron un aumento de estos receptores en células basales y secretoras del epitelio prostático durante un fotoperíodo adecuado para la especie (Bilinska et al., 1996; Saunders et al., 1996).

Por otra parte, la variabilidad en la altura del epitelio detectada dentro de las zonas glandulares, así como también en la reacción de PAS en la ZA, podría estar reflejando un patrón secretorio distinto o diferentes estadios del ciclo secretor, lo que coincide con lo informado por Hayashi et al.), quienes, al estudiar el lóbulo prostático anterior de ratas, encontraron que la altura del epitelio 
variaba dentro de la glándula, lo mismo que la actividad secretoria. Nuestros resultados también son similares a lo informado por Vásquez \& del Sol para la reacción de PAS de la porción de la próstata del complejo prostático del conejo (Oryctolagus cuniculus), sin embargo, discrepamos frente a la reacción con Azul de Alcian, que en nuestro trabajo siempre fue negativa en ambas zonas, indicando la ausencia de glicoproteínas ácidas y sulfatadas en la secreción, a diferencia de lo informado por estos autores, quienes detectaron reacción positiva en la porción prostática a pH 2,5.

Por otra parte, la menor intensidad de la reacción de PAS, observada sólo en la zona A, en los meses de otoño/invierno, en comparación con primavera/verano, podría deberse a la mayor actividad reproductiva descrita para la especie entre abril y agosto (Adaro et al., 1999; Cepeda et al.), lo que aumentaría la evacuación de la secreción contenida en los alvéolos glandulares, siendo por esto, más difícil de detectarla. Esto concuerda con lo propuesto por Pearse (1985), en el sentido de que la cantidad de color desarrollada por la reacción, depende de la concentración de sustrato presente en el tejido. El control con diastasa salival no produjo cambios en la intensidad de la reacción, descartando la presencia de glicógeno en la secreción.

Al igual que la altura del epitelio, el grosor de la pared de ambas zonas prostáticas presentó los promedios mensuales máximos en el periodo junio-agosto, y los promedios mensuales mínimos entre los meses de diciembre y febrero, lo que se debería a la influencia de la testosterona sobre la musculatura lisa que rodea los adenómeros prostáticos, hormona que, como ya se mencionó, presenta un comportamiento similar en sus niveles plasmáticos. La testosterona actuaría sobre el músculo liso a través de RA, los que, en el tejido prostático, están presentes en todas las células epiteliales secretoras, en un porcentaje variable de células estromales y también en las células musculares lisas (Prins \& Birch), y permitiría explicar el que ante la deprivación de andrógenos se produzca una disminución de la actividad secretoria de los órganos reproductivos y la atrofia de los adenómeros glandulares con disminución en la altura del epitelio (Carballada et al.).

Otros estudios, han mostrado que las células musculares lisas prostáticas son uniformemente positivas para RA y son capaces de regular la expresión de factores de crecimiento prostático. En la próstata del perro, estas células caen en atrofia y apoptosis luego de la castración, lo que junto a los cambios que sufre el epitelio glandular, indicaría que la glándula completa es un órgano sensible a los andrógenos (Niu et al.).

\section{CONCLUSIONES}

Nuestros resultados permitirían postular, que estos animales en cautiverio mantendrían la misma ciclicidad en la actividad reproductiva descrita para las chinchillas silvestres (Neira), donde las horas luz son las que, fundamentalmente, regulan el inicio y término de la temporada de apareamiento, produciendo variaciones en la concentración de testosterona sanguínea que gatillarían los cambios estructurales y funcionales de la próstata.

CEPEDA, C. R.; ADARO, A. L. \& PEÑAILILLO, G. P. Morphometric variations of Chinchilla laniger prostate and plasmatic testosterone concentration during its annual reproductive cycle. Int. J. Morphol., 24(1):89-97, 2006.

SUMMARY: The prostate of adult individuals of the species Chinchilla laniger (MOLINA, 1982) kept in captivity was studied during a one year period, in order to detect possible seasonal variations in its glandular and corporal weight, organo-somatic index (OSI), wall thickness and glandular epithelium height, and in plasmatic testosterone concentration. In addition, a histochemical analysis was performed with PAS and diastase-PAS and Blue of Alcian $\mathrm{pH} 1$ and $\mathrm{pH} 2.5$.

A periphery Zone A and a central Zone B are described. In both zones the epithelial cells always gave negative results to Blue of Alcian. The presence of neutral glicoproteins was detected in Zone A, with high PAS (+++) reaction between October and April and slight to moderate $(+/++)$ between May and September, and absence of glycogen in all cases. The B zone gave always negative PAS. The maximum morphometric values were recorded between May and July, while the minimum values were recorded in November and February, showing significant seasonal differences $(\mathrm{p} \leq 0.05)$, excepting of corporal weight, which did not show variations during the one year period.

The concentration of plasmatic testosterone showed minimum values in January, February and June, and maximum values in April, May, July, and August.

KEY WORDS: Prostate; Chinchilla laniger; Reproductive seasonal; Rodent. 


\section{REFERENCIAS BIBLIOGRÁFICAS}

Adaro, L.; Oróstegui, C.; Olivares, R. \& Villanueva, S. Variaciones morfométricas anuales del sistema reproductor masculino de la chinchilla en cautiverio (Chinchilla lanigera GREY). Avances en Producción Animal, 24(12):91-5, 1999.

Adaro, L.; Parraguez, V. H.; Oróstegui, C.; Urquieta, B. \& Cepeda, C. Variación anual de la concentración de testosterona plasmática en Chinchilla laniger (GREY) en cautiverio. Avances en Ciencias Veterinarias, 17(12):25-8, 2002.

Ayala, A; Ro, J.; Babaian, R.; Troncoso, P. \& Grignon, D. The prostatic capsule: does it exist? Its importance in the stagin and treatment of prostatic carcinoma. Am.J. of Surg. Pathol., 13(1):21-7, 1989.

Bilinska, B.; Slomczynska, M. \& Kmicikiewicz, I. Immunocytochemical demonstration of androgen receptors in Leydig cells of the bank vole (Clethrionomys glareolus, Schrober ): An in vitro study. Acta Histochemica, 98:157-64, 1996.

Branch, L. Seasonal patterns of activity and body mass in the plains vizcacha, Lagostomus maximus (familia Chinchillidae). Canadian J. of Zoology, 71:1041-5, 1993.

Campos, H. Mamíferos terrestres de Chile. Guía de Reconocimiento. Edit. Marisa Cuneo, Valdivia, Chile, 1996. pp 222.

Carballada, R. \& Bustos-Obregón, E; Esponda, P. Photoperiod-induced changes in the proteins secreted by the male genital tract of the rodent Octodon degus. J. of Experimental Zoology, 272:384-94, 1995.

Cepeda, R.; Peñailillo, P.; Urquieta, B. \& Oróstegui, C. Variaciones estacionales de las glándulas bulbouretrales de la Chinchilla lanígera en cautiverio. Aspectos morfológicos y endocrinos. In: X Congreso Nacional de Medicina Veterinaria. Valdivia 1-4 abril, 1999. pp.113-114.

Cofré, H. \& Marquet, P.A. Conservation status, rarity and geographic priorities for conservation of Chilean mammals: an assessment. Biological Conservation, 88: 53-68, 1999.

Contreras, L. \& Bustos-Obregón, E. Ciclo reproductivo anual en Octodon degus (Molina) macho. Medio Ambiente, 3(1):83-90, 1977.
Dellmann, V. Histología Veterinaria. $2^{\mathrm{a}}$ ed. Acribia, Zaragoza, 1993. pp 257-60.

García, X.; Neira, R. \& Scheu, R. Variación ambiental en características reproductivas en chinchillas (Chinchilla laniger Grey) en confinamiento. Avances en Producción Animal, 14(1-2):121-7, 1989.

Glade, A. Libro rojo de los vertebrados chilenos. CONAF, Ministerio de Agricultura. Santiago, Chile, 1988. 65 pp.

Hayashi, N.; Sugimura, Y.; Kawamura, J.; Donjacour, A. \& Cunha, G. Morphological and functional heterogeneity in the rat prostatic gland. Biology of Reproduction, 45: 308-21, 1991.

Heinlein, C. \& Chang, CH. Androgen receptor in prostate cancer. Endocrine Reviews, 25(2):276-308, 2004.

Holtz,W.; Foote, H. The anatomy of the reproductive system in male dutch rabbits (Oryctolagus cuniculus) with special emphasis on the accessory sex glands. $J$. Morphol., 156:1-12, 1978.

Jiménez, J. Conservation of the last wild chinchilla (Chinchilla laniger) archipelago: a metapopulation approach. Vida Silvestre Neotropical, 4: 89-97, 1995.

Kinbara, H.; Cunha, G. Ductal heterogeneity in rat dorsallateral prostate. The Prostate, 28:58-64,1996.

López, M.; Leyton, C.; Graf, M. Técnicas de Histología y Citología. 2. ed. Depto. de Biología Celular y Genética. Facultad de Medicina, Universidad de Chile, 1982.

Marinovic, S. Como criar chinchillas. Nociones básicas. Chile Agrícola, 15(156):150-1, 1990.

Martinelli, P. M.; Nogueira, J. C. \& Campos, P. A. Morfología e histoquímica de glicogênio e mucossubstâncias na próstata e glándulas bulbo-uretrais de Marnosa cinera Temminck, 1824 (Marsupialia-Didelphidea). Rev. bras. Ciên. Morfol., 7/8(2/1):3-12. 1991.

Miller, M.E.; Christensen, G. C. \& Evans H. E. Anatomy of the dog. W. B. Saunders Company, Philadelphia, 1965.

Moussa, M.; Badawy, A.; Kandil, M. \&Shahin, Y. Histological and histochemical studies of the accessory genital glands of bufalo-bull (Bubalis bubalis). Anat. Anz., 153:429-39, 1983. 
Murakoshi, M.; Tagawa, M.; Inada, R. \& Suzuki, M. Effects of testosterone, and testosterone plus estrogen, in the castrated rat ventral prostate - Histopathological and Immunocytochemical studies. Tokai J. Experimental and Clinical Medicine, 17 (3,4):133-7, 1992.

Murakoshi, M.; Tagawa, M.; Inada, R.; Mizokami, A.; Suzuki, M. \& Watanabe, K. Immunolocalization of glutathione-peroxidase and androgen receptors in the rat dorsolateral prostate. Tokai J. Experimental and Clinical Medicine, 18(3, 4, 5, 6 ): 87-94, 1993.

Neira, R. Chinchilla: explotación y perspectivas de desarrollo en Chile. Próxima Década, 59: 4-6, 1987.

Neira, R.; García, X. \& Scheu, R. Análisis descriptivo del comportamiento reproductivo y de crecimiento de chinchillas (Chinchilla laniger Grey) en confinamiento. Avances en Producción Animal, 14 (1-2):109-19, 1989.

Niu, Y. J.; Ma, T. X.; Zhang, J.; Xu, Y.; Han, R. F.; Sun, G. Androgen and prostatic stroma. Asian J. Andrology, 5: 19-26, 2003.

Orgad, U.; Alroy, J.; Ucci, A.; Merk, F. Histochemical studies of epithelial cell glycoconjugates in atrophic, metaplastic, hyperplastic, and neoplastic canine prostate. Laboratory Investigation, 50 (3):294-302, 1984.

Parodi, O. La Chinchilla. Editorial Albatros, Buenos Aires, 1990. 98 pp.

Pearse, A. Histochemistry theoretical and applied. $4^{\mathrm{a}}$ ed. Editorial J. \& A. Churchill Livingstone, Londres, 1985. pp 675-86.

Prins, G. \& Birch, L. Immunocytochemical analysis of androgen receptor along the ducts of the separate rat prostate lobes after androgen withdrawal and replacement. Endocrinology, 132(1):169-78. 1993.

Reighardt, J. \& Jennings, H.S. Anatomy of the cat. Holt, Rinehart and Winston. New York, Chicago-San Francisco, Toronto-London.Third and enlarged ed. 1966.

Rodríguez, J. Crianza de chinchillas. El Campesino, 26-40, 1988.

Saunders, P.; Millar, M.; Majdic, G.; Bremner, W.; Mclaren, T.; Grigor, K.; Sharpe, R. Testicular androgen receptor protein: Distribution and control of expression. In: Serono Symposia Norwell, Massachusetts, USA, 1996. pp 213-29.
SAG (SERVICIO AGRÍCOLA Y GANADERO). Estadísticas (1979-1995) de comercio exterior de productos silvoagropecuarios de Chile, Santiago, 1996. 30 pp.

Sattar, A.; Nöel, J.; Vanderhaeghen, J.; Schulman, C. \& Wespes, E. Prostate capsule: computerized morphometric analysis of its components. Urology, 46(2):178-81. 1995.

Spotorno, A.; Walker, L. Origen y evolución de los mamíferos chilenos. In: Muñoz, P. A. \& Yáñez, J. (Eds.). Mamíferos de Chile. Editorial CEA, Valdivia, Chile, 2000. pp 217-227.

Spotorno, A.; Zuleta, C.; Valladares, J.; Deane, A. L. \& Jiménez, J. Chinchilla lanigera. Mammalian species, 758:1-19, 2004.

Thornback, J.; Jenkis, M. The IUCN mammal red data book. Part: Threatened mammalian taxa of the Americas and the Austrasia zoogeographic region (excluding Cetacea). International Union Conservation Natural Gland, Switzerland, 1982. $516 \mathrm{pp}$.

Vanha-Pertula, T.; Ronkko, S. \& Lahtinen, R. Hydrolases from bovine seminal vesicle, prostate and Cowper's gland. Andrología, 22(1):10-24. 1990.

Vásquez, B. \& del Sol, M. Complejo prostático en el conejo (Oryctolagus cuniculus). Rev. Chil. Anat., 20(2):175-80. 2002.

Weir, B. The chinchilla. The UFAW handbook on the care and management of laboratory animals. 4 ed. Livingston, Edinburgh, 1972. pp 269-277.

Dirección para correspondencia:

Prof. Dra. Raquel Cepeda

Universidad de Chile

Facultad de Ciencias Veterinarias y Pecuarias

Departamento de Ciencias Biologicas Animales

Santiago - CHILE

Email:rcepeda@uchile.cl

Recibido : 22-08-2005

Aceptado: 12-01-2006 